typeset using JPSJ.sty $<$ ver.1.0b $>$

\title{
Monte Carlo Study of the Separation of Energy Scales in Quantum Spin 1/2 Chains with Bond Disorder
}

\author{
Beat Ammont and Manfred Sigrist ${ }^{1, * *}$ \\ SCSC and Theoretische Physik, Eidgenössische Technische Hochschule, CH-8092 Zürich, Switzerland \\ ${ }^{1}$ Yukawa Institute for Theoretical Physics, Kyoto University, Kyoto 606-8502
}

(Received )

\begin{abstract}
One-dimensional Heisenberg spin 1/2 chains with random ferro- and antiferromagnetic bonds are realized in systems such as $\mathrm{Sr}_{3} \mathrm{CuPt}_{1-x} \mathrm{Ir}_{x} \mathrm{O}_{6}$. We have investigated numerically the thermodynamic properties of a generic random bond model and of a realistic model of $\mathrm{Sr}_{3} \mathrm{CuPt}_{1-x} \mathrm{Ir}_{x} \mathrm{O}_{6}$ by the quantum Monte Carlo loop algorithm. For the first time we demonstrate the separation into three different temperature regimes for the original Hamiltonian based on an exact treatment, especially we show that the intermediate temperature regime is well-defined and observable in both the specific heat and the magnetic susceptibility. The crossover between the regimes is indicated by peaks in the specific heat. The uniform magnetic susceptibility shows Curie-like behavior in the high-, intermediate- and low-temperature regime, with different values of the Curie constant in each regime. We show that these regimes are overlapping in the realistic model and give numerical data for the analysis of experimental tests.
\end{abstract}

KEYWORDS: quasi-one-dimensional spin system, random spin chain, quantum Monte Carlo, $\mathrm{Sr}_{3} \mathrm{CuPt}_{1-x} \operatorname{Ir}_{x} \mathrm{O}_{6}$

\section{$\S 1$. Introduction}

One-dimensional quantum spin chains are typical examples of many-body systems with a very rich variety of physical properties. Over many decades they have attracted much interest in theory and have motivated the development of various calculation schemes, both analytical and numerical. The number of real compounds containing quasi-one-dimensional spin systems is growing. A few examples are organic systems like NENP and NINOHE or inorganic compounds such as $\mathrm{Sr}_{3} \mathrm{MPtO}_{6}$ (M $=\mathrm{Ni}, \mathrm{Cu}$ and $\mathrm{Zn}$ ). $\mathrm{B}^{\mathrm{C}}$ Some of them are investigated as possible realizations of Haldane gap systems. Recently also ladder systems such as $(\mathrm{Ca})_{2} \mathrm{VO}_{3}$ and $\mathrm{Sr}_{2} \mathrm{CuO}_{3}$ were investigated as an example of a resonating valence bond system. All these systems are supposed to be regular chains or ladders. However, in practice disorder must occur in most of these systems. Already little disorder in the composition can have considerable influence on the low-energy properties. A peculiar example of a disordered spin chain was recently discovered by Nguyen and zur Loye: the alloy $\mathrm{Sr}_{3} \mathrm{CuPt}_{1-x} \mathrm{Ir}_{x} \mathrm{O}_{6}$ (-1) The pure compound $\mathrm{Sr}_{3} \mathrm{CuPtO}_{6}$ forms an antiferromagnetic (AF) spin chain where a spin $1 / 2$ is provided by each $\mathrm{Cu}$-ion. In this compound the $\mathrm{Cu}$-ions are alternating with the spinless Pt-ions along chains. If all $\mathrm{Pt}$ is replaced by Ir which carries a spin $1 / 2$, then the system is a ferromagnetic (FM) spin chain. Therefore the random alloy $\mathrm{Sr}_{3} \mathrm{CuPt}_{1-x} \mathrm{Ir}_{x} \mathrm{O}_{6}$ is a spin system with two types of bonds, FM $\left(J_{\mathrm{F}}<0\right)$ and $\mathrm{AF}\left(J_{\mathrm{A}}>0\right)$, which are randomly distributed. There is a correlation among the

\footnotetext{
* Present address: Institute for Solid State Physics, University of Tokyo, Roppongi 7-22-1, Tokyo 106. E-mail: ammon@ginnan.issp.u-tokyo.ac.jp

** E-mail: sigrist@yukawa.kyoto-u.ac.jp
}

bonds in the sense that FM bonds always occur in sequences of even numbers, since each Ir-ion makes such bonds with its two neighboring $\mathrm{Cu}$-ions. A generic model to study the properties of such a system was considered by Furusaki and coworkers 5 who neglected the correlation among the bonds and used $J=\left|J_{\mathrm{F}}\right|=\left|J_{\mathrm{A}}\right|$. The quantum spin system in their analysis has the following nearest neighbor Heisenberg Hamiltonian

$$
\mathcal{H}=\sum_{i} J_{i} \mathbf{S}_{i} \cdot \mathbf{S}_{i+1},
$$

with a bond probability distribution which is independent of $i$

$$
P\left(J_{i}\right)=p \delta\left(J_{i}+J_{\mathrm{F}}\right)+(1-p) \delta\left(J_{i}-J_{\mathrm{A}}\right),
$$

where $0 \leq p \leq 1$, and $\delta$ is the Kronecker delta function. For $p=0$ and 1 we have a purely AF or FM spin chain, respectively.

The analysis of this model by high-temperature expansion $\bar{E}$ and by a real space renormalization group (RSRG) scheme 6 suggests that three different temperature regimes are present in this system. In the hightemperature regime the spins essentially behave as independent degrees of freedom. If the temperature is lowered down to $k_{\mathrm{B}} T \sim J$ the spins start to correlate. First they align within the segments of purely FM or AF bonds. The coupling among the segments is weak. Thus, the spins in each segment create an effective single spin degree of freedom which is rather large in the case of FM bonds and $S=0$ or $1 / 2$ for AF bonds. At intermediate temperatures these effective spins behave as independent due to the thermal fluctuations. However, they begin to correlate at lower temperatures. The intermediate and low-temperature regimes are described by a random spin model with a basically continuous random bond distri- 
bution as well as random spin sizes (effective spin sizes).

The limit of $\mathrm{T} \rightarrow 0$ was analyzed by Westerberg and coworkers by means of a RSRG scheme for this type of model 6, Here we will briefly review their main results. As the temperature $T$ is lowered, a growing number of spins is correlated in clusters consisting of $n$ spins on the average, which form a large spin $S$. The spin size $S$ scales with $S \propto n^{1 / 2}$ and the average number of spins in a cluster scales with the temperature as $n \propto T^{-2 \alpha}$. The scaling exponent $\alpha \approx 0.22$ was determined numerically. 6. 8) Because the large effective spins $S$ of the clusters behave as essentially independent under thermal fluctuations, one expects a Curie-like susceptibility $\chi \propto C / T$ with a $T$ dependent Curie constant $C$.

From this result we conclude that, in principle, this system exhibits three different temperature regimes, each with its own Curie-like susceptibility and Curie constant. In the high-temperature regime $\left(k_{\mathrm{B}} T \gg J\right)$ the Curiebehavior originates from the $S=1 / 2$ spins leading to $\chi=\frac{\mu_{\mathrm{B}}^{2} C}{k_{\mathrm{B}} T}$, where $\mu_{\mathrm{B}}=\frac{e \hbar}{2 m c}$ is the Bohr-magneton and the Curie constant is $C=S(S+1) / 3=1 / 4$. In the intermediate temperature regime $\left(k_{\mathrm{B}} T \sim J\right)$ the effective spins of the segments give a $p$-dependent Curie constant $C_{\text {eff }}$. Finally a crossover to a scaling regime with a new Curie behavior occurs at very low-temperatures. Obviously the three Curie constants should be a decreasing sequence as the number of available degrees of freedom is decreasing with lowering temperature.

The separation into three regimes is expected to be visible in the specific heat too. Peak- or shoulder-like structures indicate the boundaries between the regimes as they are sign of correlation of degrees of freedom. One boundary occurs near $k_{\mathrm{B}} T \sim J$ and the second at some lower temperature. In the scaling regime at very low temperatures, the assumption of independent large spins $S$ of correlated clusters leads to $\left.c_{\mathrm{V}} / T \propto T^{2 \alpha-1}|\ln T| 6\right)$

The crossover between the high- and intermediate temperature regime has been examined by the hightemperature expansion. For low temperatures, however, only the limiting behavior for $T \rightarrow 0$ has been investigated for an effective Hamiltonian with a broad random distribution of couplings $J_{i}$, 8 , which is in contrast to the discrete distribution of the initial spin couplings in $\mathrm{Sr}_{3} \mathrm{CuPt}_{1-x} \mathrm{Ir}_{x} \mathrm{O}_{6}$. From these results we know that the effective spin scaling regime starts at very low temperatures, which are hard to observe by experiment. The actual crossover from the intermediate to the lowtemperature regime can be investigated more easily by experiment, but has not yet been analyzed so far. In this paper we would like to close this gap by demonstrating that the intermediate temperature regime is well-defined and observable in both the specific heat and the susceptibility by an exact treatment of the original Hamiltonian.

The correlations among the FM bonds in $\mathrm{Sr}_{3} \mathrm{CuPt}_{1-x} \mathrm{Ir}_{x} \mathrm{O}_{6}$ are another important aspect which has not yet been examined. We will investigate the implications of these correlations in a realistic model where the FM bonds always occur pairwise and study its differences to the uncorrelated model in the magnetic susceptibility and the specific heat in an experimentally accessible temperature range. These data will provide a sensitive test for the randomness of the distribution of the Pt-ions in $\mathrm{Sr}_{3} \mathrm{CuPt}_{1-x} \mathrm{Ir}_{x} \mathrm{O}_{6}$.

Our investigations are based on QuantumMonte Carlo (QMC) simulations by the loop algorithm, which is an excellent method to simulate accurately the thermodynamics of large spin systems.

\section{§2. Numerical Methods}

The QMC loop algorithm9) is a finite temperature method based on the Trotter-Suzuki decomposition 10 ) of the partition function $Z$ and is ideally suited for the calculation of thermodynamic properties of unfrustrated spin systems. It allows the direct and exact computation of various thermodynamic observables, e.g. the uniform magnetic susceptibility $\chi$ or the internal energy $u$, without introducing any approximations. In contrast to the classical Metropolis world-line algorithm,11] the loop-algorithm does not suffer from prohibitively long auto-correlation times. Additionally, the introduction of improved estimators12) gives a further reduction of the variance. This enables us to investigate much bigger problems than with previous QMC methods.

For the calculation of the random bond models, we have considered up to 400 samples of different random bond configurations, each consisting of a chain of $L=$ 400 sites with periodic boundary conditions. In Fig. 1 we show the distribution of FM (AF) segments of length $l_{s}$ in our configuration samples. We have performed

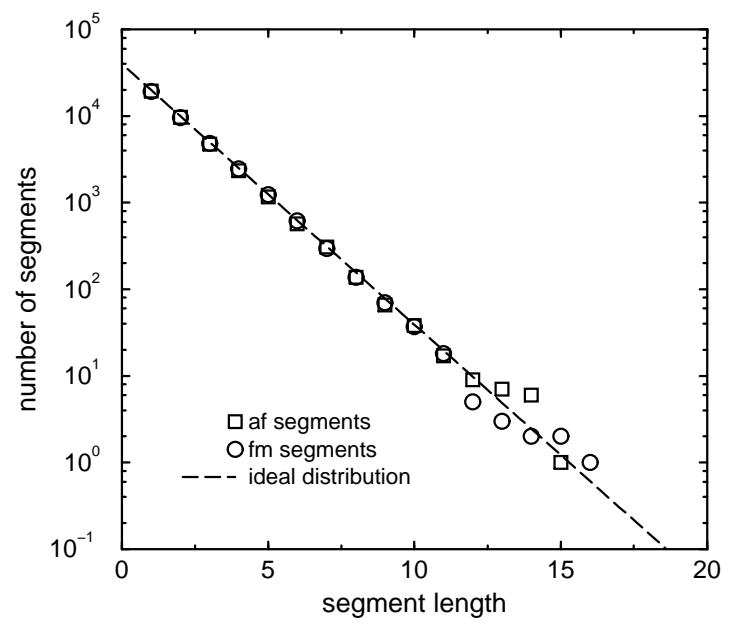

Fig. 1. Statistic of the total distribution of the segments with $l_{s}$ FM (squares) and AF (circles) bonds in the configurations we have used for the simulation of the unconstrained model. The dashed curve shows the ideal distribution.

calculations in the temperature range from $T / J=1 / 60$ to $T / J=10$. Unless otherwise mentioned, we have used Trotter numbers between 20 and 120 and extrapolated to Trotter time-steps $\Delta \tau J=0$. We made $10^{4}$ multi-cluster updates for thermalization, followed by $2 \times 10^{5}$ up to $2 \times$ $10^{6}$ multi-cluster updates for measurements, depending on the temperature $T$ and the Trotter-number. 
The value of an observable $\mathcal{O}$ is estimated by averaging over $M$ successive measurements $O_{j}$ coming from the QMC simulation: $\langle\mathcal{O}\rangle \approx \bar{O}=M^{-1} \sum_{j=1}^{M} O_{j}$, and the error is determined by the variance of the measurements. In this way we have measured directly the internal energy $u$, the total magnetization $M$, and the uniform susceptibility $\chi$ for each configuration. Due to the efficiency of our QMC algorithm, we have been able to measure the internal energy $u$ accurately enough to calculate the specific heat numerically as the first derivative of the internal energy $u$ with respect to the temperature $T$. After the calculation of all observables for each individual configuration, the average over all configurations has been taken. In order to get a correct estimate of the errors, we have taken into account the variance of the observable for the set of configurations and the error of the observable for a single configuration. In general, the error-bars for the observables of a single configuration are much smaller than the variance of the observables for the set of configurations, and therefore we can neglect them completely.

\section{$\S 3 . \quad$ Results}

We have studied two different models. First, we have investigated a generic random bond model with $\mathcal{H}=$ $\sum_{i} J_{i} \mathbf{S}_{i} \cdot \mathbf{S}_{i+1}$ and the bond probability distribution

$$
P\left(J_{i}\right)=p \delta\left(J_{i}+J_{\mathrm{F}}\right)+(1-p) \delta\left(J_{i}-J_{\mathrm{A}}\right),
$$

where $0 \leq p \leq 1$ and $\left|J_{\mathrm{F}}\right|=\left|J_{\mathrm{A}}\right|$ for the case of $p=0.5$, i.e., the same probability for finding a FM as an AF bond. This model has been studied in a number of papers using different methods.5, 6, In the rest of this paper we will call this model the "unconstrained" model.

In the real $\mathrm{Sr}_{3} \mathrm{CuPt}_{1-x} \mathrm{Ir}_{x} \mathrm{O}_{6}$ alloys, there are some additional restrictions. As we have previously mentioned in the introduction, by replacing the spinless $\mathrm{Pt}$-ions with Ir-ions, two FM bonds are created between the spin 1/2 carrying Ir-ions and its neighboring $\mathrm{Cu}$-ions. In addition to this pairwise correlation of FM bonds, the FM couplings are stronger than the AF couplings, an estimate gives $\left|J_{\mathrm{F}}\right|=4\left|J_{\mathrm{A}}\right|$. By generating a random sequence of Pt- an Ir-ions we have generated a configurations equivalent to and Ir substitution of $x=0.5$, which corresponds to the probability of finding a FM bond with $p=2 / 3$. We will refer to these systems as the "constrained" model in the following text of this paper.

\section{$\S 4$. Susceptibility}

Let us consider the unconstrained model first. In the high-temperature regime $k_{\mathrm{B}} T \gg J$, we have independent single spins as in the uniform AF or FM chain, and a Curie-law for the susceptibility $\chi_{\mathrm{cl}}(T)=\mu^{2} /\left(4 k_{\mathrm{B}} T\right)$ for $T \rightarrow \infty$. As the temperature $T$ is lowered, the individual spins start to correlate. In the AF segments, they form the collectively lowest possible total spin $(S=0$ for an odd number of bonds $l_{s}$ or $S=1 / 2$ for an even number $l_{s}$ ), while the FM segments form the collectively largest spin (by aligning all spins parallel). Due to the misfit of the discrete spectra in the AF/FM spin segments (the finite size gap $\delta E_{\mathrm{FS}}$ is $\delta E_{\mathrm{FS}} \propto J / l_{s}$ for $\mathrm{AF}$ and $\delta E_{\mathrm{FS}} \propto J / l_{s}^{2}$ for FM segments), the excitations remain localized in the segments and the interactions among different segments remain very weak. In this intermediate temperature regime the segments essentially behave as uncoupled effective spins $S_{\text {eff }}$. These uncoupled effective spins can be seen in a second Curie-law of the susceptibility $\chi$ on an intermediate energy-scale $\left(\propto \delta E_{\mathrm{FS}}\right)$ with an effective Curie-constant $C_{\text {eff }}$ depending on the average size of the effective spins

$$
\chi=\frac{\mu^{2} C_{\mathrm{eff}}}{k_{\mathrm{B}} T},
$$

with $C_{\text {eff }}=\frac{1}{3} \frac{\left\langle S_{\mathrm{FM}}^{2}\right\rangle+\left\langle S_{\mathrm{AF}}^{2}\right\rangle}{\left\langle n_{\mathrm{FM}}\right\rangle+\left\langle n_{\mathrm{AF}}\right\rangle}$, where $\left\langle S_{\mathrm{FM} / \mathrm{AF}}\right\rangle$ denotes the effective spin of the FM/AF segment, and $\left\langle n_{\mathrm{FM} / \mathrm{AF}}\right\rangle$ is the average length of a FM/AF segment. The spins on the boundary of a segment of pairs of FM spins and a segment of AF spins always have to be counted to the AF segment and not to the FM segment, i.e., the total spin of a FM segment with a length of $l_{s}$ bonds is $\left.S_{\text {tot }}=S\left(l_{s}-1\right) .5\right)$ For the unconstrained model with $p=0.5$ and $S=1 / 2$ one obtains $C_{\text {eff }}=1 / 8$ for a chain of infinite length.

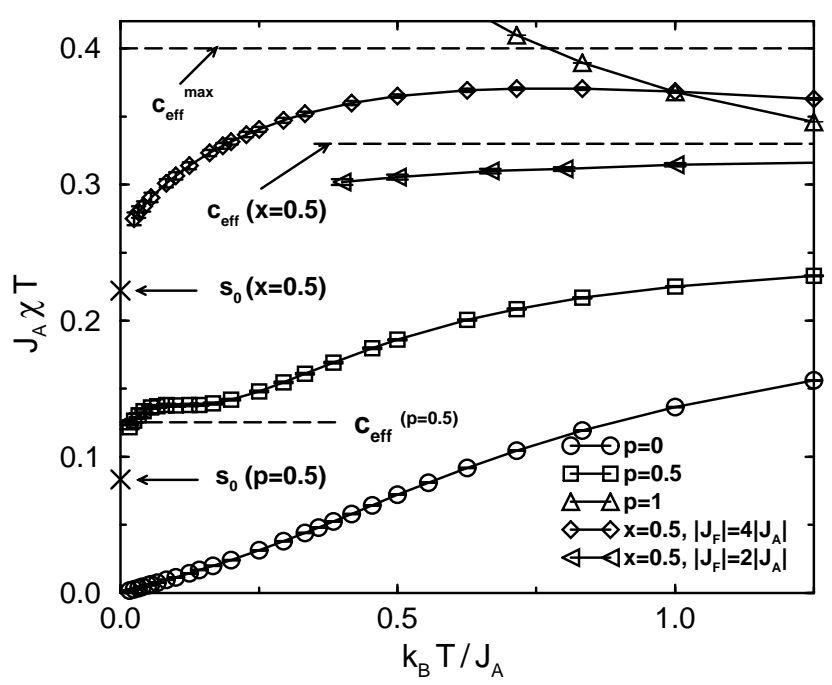

Fig. 2. Figure of the uniform magnetic susceptibility $\chi$ times temperature $T$. The symbols are the results of the QMC simulations for the unconstrained model (squares) and the constrained model of $\mathrm{Sr}_{3} \mathrm{CuPt}_{1-x} \mathrm{Ir}_{x} \mathrm{O}_{6}$ (diamonds). For comparison we also show the results for the uniform FM and AF Heisenberg chain. The Curie-law behavior can be seen by horizontal lines, the dashed lines show the results of the statistical analysis, $s_{0}$ are the limits of $\chi T$ for $T \rightarrow 0$.

In Fig. 2 we show $\chi J T$ as a function of the temperature, such that the Curie-law behavior of the effective spins can be seen as a plateau. From our QMC simulations we get $C_{\mathrm{eff}}=0.138 \pm 0.003$, whereas the value of the effective constant $C_{\text {eff }}$ obtained by statistical analysis of the bond distribution is $C_{\text {eff }}=0.1252$, and it is shown by a dashed line in the graph. Previous results by the transfer matrix method and high-temperature expansions obtained $C_{\text {eff }}=0.13 .5$ However, in the hightemperature expansion, a possible error stems from the 
extrapolation of the Padé approximants. There is also some range for modification by considering to which segment a spin on the border between FM and AF segments has to be counted in the statistical analysis: In general, the spins at the border between these segments tend to be included in the AF segments, but if a long AF segment is next to a short FM segment, some of these boundary spins rather couple to the FM segment, resulting in an increase of $C_{\text {eff. }}$ A detailed analysis of the bond distribution used in our QMC calculations shows that if we count the spins on the border of AF segments consisting of six and more bonds next to FM segments consisting of three and less bonds to the FM segment, we rather get $C_{\text {eff }}=0.131$ and similarly for $\mathrm{AF}$ segments consisting of five and more bonds next to FM segments consisting of three and less bonds we find $C_{\text {eff }}=0.138$ instead, in agreement with our QMC results.

The temperature range of the regime of uncoupled effective spins is determined by the extension of this Curie-law plateau, it starts at $k_{\mathrm{B}} T \approx 0.16 J$ and ends at $k_{\mathrm{B}} T \approx 0.06 J$. The region of this regime compares favorably to the simple estimate given by the finite size gap of the spin segments $\delta E_{\mathrm{FS}}$.

At very low temperatures, interactions among the effective spins become relevant and the effective spins start to correlate. These interactions are again described by a Heisenberg model with $H_{\text {eff }}=\sum_{i} J_{\text {eff }}^{i} \mathbf{S}_{\text {eff }}^{i} \cdot \mathbf{S}_{\text {eff }}^{i+1}$, where the sum $i$ is now taken over the spin segments. The couplings $J_{\text {eff }}^{i}$ are random in both sign and magnitude, and the size of the effective spins $S_{\text {eff }}$ is random as well. Following ref. 6, the distribution of the effective spins $S_{\text {eff }}^{i}$ and their interactions $J_{\text {eff }}^{i}$ is very broad. Hence two neighboring spins with the largest energy gap to their first excited state will be locked into their groundstate, consisting of their maximal $\left(J_{\text {eff }}<0\right)$ or minimal $\left(J_{\text {eff }}>0\right)$ spin state and form a new effective spin $S_{\text {eff }}$. As more and more spins freeze out, the effective spin size $S_{\text {eff }}$ scales with the number of original spins in the cluster as $S_{\text {eff }}^{i} \sim n^{1 / 2}$ (random walk). The average number of original spins in a cluster $n$ depends on the temperature $T$ and sfales with a power law $n \sim T^{-2 \alpha}$, with $\alpha=0.22 \pm 0.01$ ) consistent with the result $\alpha=0.21 \pm 0.01$ of ref. 8

In Fig. 3 we have plotted $J \chi T$ as a function of $T^{\alpha} / J$ for different values of $\alpha$. The low temperature behavior has been calculated in ref. 8 :

$$
\chi T=s_{0}+\frac{T^{\alpha}}{\gamma}+O\left(T^{3 \alpha}\right)
$$

where $s_{0}$ is the groundstate expectation value of the total spin per site

$$
s_{0}=\frac{\mu^{2}}{3 k_{\mathrm{B}} L}\left(\sum_{i=1}^{L} \delta_{i} S_{i}\right)^{2},
$$

where $\delta_{i+1}=-\delta_{i} \operatorname{sgn}\left(J_{i}\right)$ and $\delta_{1}=1$. The beginning of the low-temperature scaling regime is estimated to start at $T<0.05 J_{0}$ for the effective Hamiltonian, 8 ) where $J_{0}$ is the maximum of a broad random distribution of couplings. This distribution of couplings corresponds to the effective couplings $J_{\text {eff }}$ of the intermediate temperature

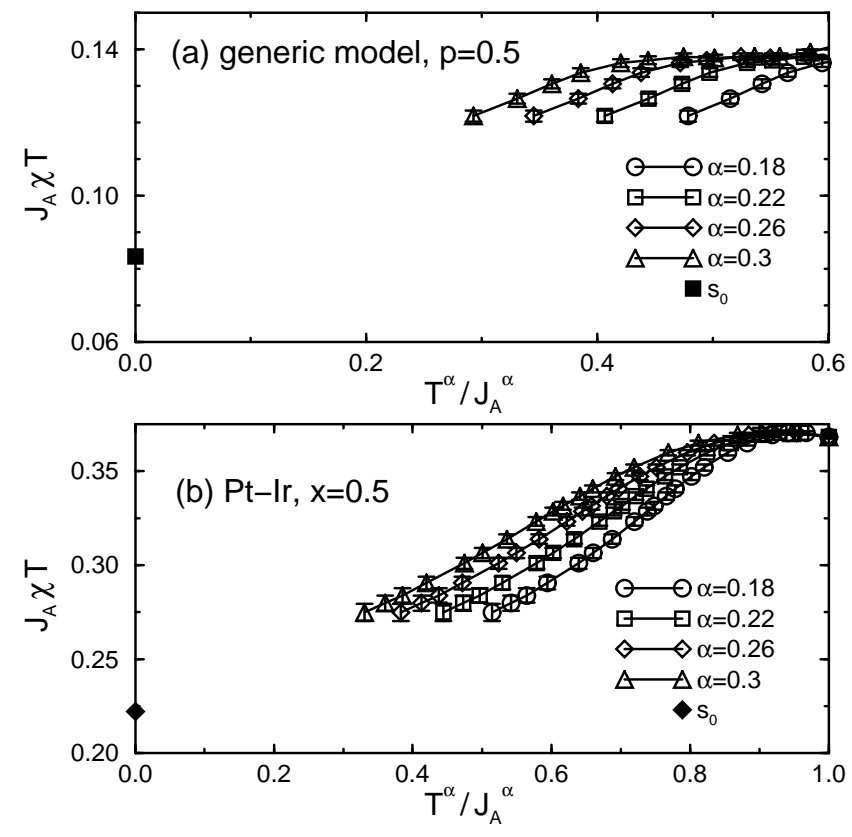

Fig. 3. Low temperature scaling behavior of $\chi T$ for the unconstrained model (a) and the constrained model (b). The lowtemperature scaling regime is characterized by $\chi T=s_{0}+T^{\alpha} / \gamma+$ $O\left(T^{3 \alpha}\right)$. The open symbols denote different values of the scaling exponent $\alpha$, the full symbols denote the $T \rightarrow 0$ limit

regime. These couplings are about an order of magnitude smaller than the original couplings 5 ) In Fig. 3, we can see that a linear extrapolation of the data points with the scaling exponent $\alpha=0.22$ does not yet yield the correct zero-temperature value $s_{0}$ and we conclude that the scaling regime has not been reached for $T=0.02 \mathrm{~J}$. Unfortunately, critical slowing down in the QMC simulation at low temperatures prevents us from reaching temperatures as low as $T<0.005 \mathrm{~J}$.

Next we concentrate on the susceptibility of the constrained model of $\mathrm{Sr}_{3} \mathrm{CuPt}_{1-x} \mathrm{Ir}_{x} \mathrm{O}_{6}$. In this case we have to take into account the different strength of the FM and $\mathrm{AF}$ interactions. Starting from the same high temperature limit $\chi / L=\frac{1}{4} J / T$ as in the generic case, the FM bonds start to correlate by aligning parallel at higher temperatures $T$ than the AF bonds, due to the stronger coupling $\left|J_{\mathrm{F}}\right|=4\left|J_{\mathrm{A}}\right|=4 J$. The formation of these large spins leads to an increase of $J \chi T$ first. Then the AF spins start to correlate too, but instead of a plateau as in the case of the unconstrained model we obtain a broad peak near $k_{\mathrm{B}} T=J$.

If we calculate the average effective spins $\left\langle S_{\mathrm{FM} / \mathrm{AF}}\right\rangle$ as in the previous case, we obtain an estimate of the effective Curie constant $C_{\text {eff }}=0.33 \pm 0.017$. This value is a lower bound of the Curie constant, where the spins within the segments are completely correlated. The effect of the constraint among the FM bonds in $\mathrm{Sr}_{3} \mathrm{CuPt}_{1-x} \operatorname{Ir}_{x} \mathrm{O}_{6}$ is very important, if we neglect that we would obtain $C_{\text {eff }} \approx 0.2731$ instead. An upper bound of $C_{\text {eff }}^{\max }=0.40 \pm 0.02$ can be obtained by assuming that only the spins in the FM segments correlate and the 
AF segments remain uncoupled due to their weaker couplings. From our QMC data in Fig. 3 we get $C_{\text {eff }} \approx 0.36$. The deviation from $C_{\text {eff }}$ of the spin segment statistic is larger than for the unconstrained model, since the difference in the coupling strength favors quantum effects on the boundaries of large FM and small AF segments and makes the simple statistical estimate more subtle. The effects causing an increase of of $C_{\text {eff }}$ are twofold in this case: the first is the same as for the unconstrained model, spins on the border of large AF segments next to a short FM segments tend to couple to the FM segment. A detailed analysis of the spin distribution gives $C_{\text {eff }}=0.34$ if we count the spins on the border of $\mathrm{AF}$ segments consisting of five and more bonds next to FM segments consisting of four and less bonds to the FM segment, and $C_{\text {eff }}=0.345$ for $\mathrm{AF}$ segments consisting of four and more bonds next to a segments consisting of three and less bonds. The second reason for a larger value of $C_{\text {eff }}$ is that due to the relatively weaker AF couplings than the FM couplings, some of the AF segments are not yet completely correlated. Thus especially the long AF segments still consist of some original spin-1/2 degrees of freedom, giving rise to a further increase of $C_{\text {eff }}$ as we have demonstrated for the case of completely uncorrelated AF segments before.

Additionally we have also determined $C_{\text {eff }}$ by a QMC simulation for the same distribution as above but with different coupling strength $\left|J_{\mathrm{F}}\right|=2\left|J_{\mathrm{A}}\right|=2 J$. Here we can see again how the susceptibility $\chi T J$ increases from the high-temperature limit $\chi T J=1 / 4$; but in contrast to the previous case it never reaches the lower bound of $C_{\text {eff }}=0.33 \pm 0.017$ of the regime of uncoupled effective spins. In this case, some spin segments already start to correlate and form a collective spin before the regime of independent effective spins is established.

In order to investigate the low-temperature scaling behavior of eq. (4.1) for the constrained model, we have to calculate $s_{0}$ from eq. 4.2). Let us consider a chain of $L \mathrm{Cu}$ - ions. There are $L x$ spin $1 / 2$ carrying Ir-ions in the chain and the ground state expectation value of the total spin is: $\left\langle\left(\sum_{\text {spins }} S_{i}\right)^{2}\right\rangle=$ $(1+x) L\left\langle S_{i}^{2}\right\rangle+\sum_{\text {spins }}\left\langle S_{i} S_{j}\right\rangle$, where the sum is taken over all $\mathrm{Cu}$ - and Ir-ions. For the evaluation of $\sum_{\text {spins }}\left\langle S_{i} S_{j}\right\rangle$ we divide the sum into four terms using the following notation: we enumerate all $\mathrm{Cu}$-ions with the index $i$, while we use the index $\hat{i}$ for the Ir-ion between the $\mathrm{Cu}$-ions $i$ and $i+1$ and write $\sum_{\text {spins }}\left\langle S_{i} S_{j}\right\rangle=$ $2\left(\sum_{i<j}\left\langle S_{i} S_{j}\right\rangle+\sum_{i<\hat{j}}\left\langle S_{i} S_{\hat{j}}\right\rangle+\sum_{\hat{i}<j}\left\langle S_{\hat{i}} S_{j}\right\rangle\right.$

$\left.+\sum_{\hat{i}<\hat{j}}\left\langle S_{\hat{i}} S_{\hat{j}}\right\rangle\right)$. Now we can calculate the expectation value $\left\langle S_{i} S_{j}\right\rangle$ by taking into account that an Ir-ion exists only with the probability $x$ and that each missing Ir-ion changes the sign of the next spin:

$$
\begin{aligned}
\left\langle S_{i} S_{i+n}\right\rangle & =\frac{1}{4} \sum_{m=0}^{n} x^{m}(1-x)^{n-m}(-1)^{n-m}\left(\begin{array}{c}
n \\
m
\end{array}\right) \\
& =\frac{1}{4}(2 x-1)^{n}
\end{aligned}
$$

$$
\begin{aligned}
\left\langle S_{i} S_{\widehat{i+n}}\right\rangle & =\frac{x}{4} \sum_{m=0}^{n} x^{m}(1-x)^{n-m}(-1)^{n-m}\left(\begin{array}{c}
n \\
m
\end{array}\right) \\
& =\frac{x}{4}(2 x-1)^{n} \\
\left\langle S_{\hat{i}} S_{i+n}\right\rangle & =\frac{1}{4} \sum_{m=0}^{n-1} x^{m}(1-x)^{n-1-m}(-1)^{n-1-m}\left(\begin{array}{c}
n-1 \\
m
\end{array}\right) \\
& =\frac{1}{4}(2 x-1)^{n-1} \\
\left\langle S_{\hat{i}} S_{\widehat{i+n}}\right\rangle & =\frac{x}{4} \sum_{m=0}^{n-1} x^{m}(1-x)^{n-1-m}(-1)^{n-1-m}\left(\begin{array}{c}
n \\
m
\end{array}\right) \\
& =\frac{x}{4}(2 x-1)^{n-1},
\end{aligned}
$$

where $\left(\begin{array}{c}n \\ m\end{array}\right)=n ! /(m !(n-m) !)$. From this we obtain

$$
\begin{aligned}
& \left\langle\left(\sum_{\text {spins }} S_{i}\right)^{2}\right\rangle \\
& \frac{2 L}{4}\left[\sum_{n=1}^{L}(2 x-1)^{n}+\sum_{n=0}^{L} x(2 x-1)^{n}\right. \\
& +x \\
& \left.\left(\sum_{n=1}^{L}(2 x-1)^{n-1}+\sum_{n=1}^{L} x(2 x-1)^{n-1}\right)\right] \\
& =L,
\end{aligned}
$$

which gives in the groundstate $s_{0}=\left\langle\left(\sum_{\text {spins }} S_{i}\right)^{2}\right\rangle /\left(3 k_{\mathrm{B}}(1+x) L\right)=2 / 9 \approx 0.222222 \ldots$.

In Fig. 3 we can see that also for this model the value we get for $s_{0}$ is too small if we extrapolate our data linearly with the correct scaling exponent $\alpha \approx 0.22$. Thus we conclude that the low-temperature scaling regime starts below $T / J_{\mathrm{A}}<1 / 100$.

\section{§5. Specific Heat}

Again, let us concentrate on the unconstrained model first. In the specific heat data, the crossover between different temperature regimes should be visible by peaklike structures, where the spins start to correlate. In the uniform FM or AF Heisenberg systems, one broad peak below $k_{\mathrm{B}} T / J \sim 1$ appears, where the individual spins start to correlate. Two peaks are expected in the random system. One where the original $S=1 / 2$ spins start to correlate (as for the uniform systems), and a second one where the segments of the effective spins start to correlate (at the end of the Curie-plateau $k_{\mathrm{B}} T / J \sim 1 / 20$ ). In Fig. 4 we show the specific heat per spin $c_{\mathrm{V}}$ of the random bond systems and of the uniform AF and FM chain for comparison. The large and broad peak is the signature of the correlation of the individual spins. In the inset, one can clearly see the second peak at low temperatures of the correlation of the effective spins for the unconstrained model. This confirms the clear separation of the different regimes and could not be investigated with the previous methods, e.g. the high-temperature expansion.

In the scaling regime at very low temperatures $T$, the assumption of independent large spins $S$ of cor- 


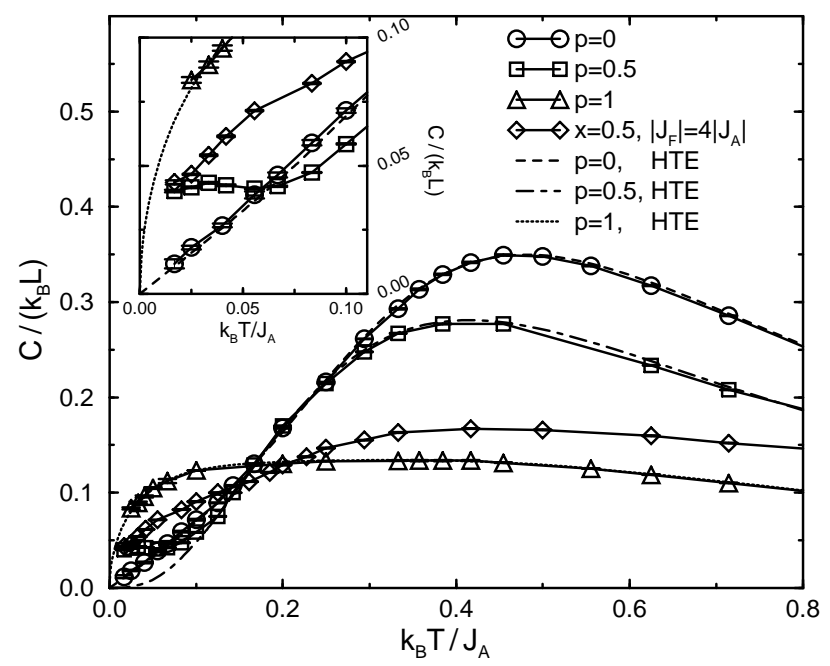

Fig. 4. The specific heat per spin $c_{\mathrm{V}}$ of the unconstrained random bond model and the $\mathrm{Sr}_{3} \mathrm{CuPt}_{1-x} \mathrm{Ir}_{x} \mathrm{O}_{6}$-model. For comparison, we have also shown the results of the uniform FM and AF Heisenberg chain. The symbols are the results of the QMC simulation for the generic model (squares) and the constrained model of $\mathrm{Sr}_{3} \mathrm{CuPt}_{1-x} \mathrm{Ir}_{x} \mathrm{O}_{6}$ (diamonds), dashed and dotted lines (HTE) are the high-temperature expansion results obtained by the extrapolation of the two-point Padé approximants. In the inset, one can see the peak in the specific heat of the unconstrained model and a cusp for the constrained model which indicate the onset of correlations among the effective spins.

related clusters 5 leads to the entropy per site $\sigma \propto$ $\ln (2 S(T)+1) / n(T) \propto T^{-2 \alpha}|\ln T|$. From this one finds $c_{\mathrm{V}} / T \propto T^{-2 \alpha-1}|\ln T|$. But as for the magnetic susceptibility, we can not reach such low temperatures as to determine the scaling exponent $\alpha$ from our data. By the relation $\int_{0}^{T} \mathrm{~d} T c_{\mathrm{V}} / T$, we can see from the divergence of $c_{\mathrm{V}} / T$ as $T \rightarrow 0$ that a large fraction of the entropy is at very low temperatures, due to the broad spectrum of energy-scales of the effective spins. In Fig. 5 we show $c_{\mathrm{V}} / T$, the area below the curve corresponds to the entropy $S$. If we consider the data in Fig. 5 in more detail, we can see that the value of $c_{\mathrm{V}} / T$ for the generic model is always smaller than $c_{\mathrm{V}} / T$ of the uniform AF Heisenberg chain. Only at very low temperatures, we can see a sharp crossover to the divergent low-temperature behavior. This crossover is due to the very different energyscales of the effective spins and can not be seen by the high-temperature expansion.

More important for comparisons with experiments is the specific heat of the constrained model of $\mathrm{Sr}_{3} \mathrm{CuPt}_{1-x} \operatorname{Ir}_{x} \mathrm{O}_{6}$. Here, the spins start to correlate at different temperatures $T / J$ due to the different coupling strengths. Hence there is no clear transition and the peak at $T / J \approx 1$ is much weaker than for the unconstrained model. Also the peak at the transition to the scaling regime is much weaker, but we can interpreted the cusplike structure near $T \approx 0.05 J$ as the corresponding peak in the specific heat. In Fig. 5 , where we show $c_{\mathrm{V}} / T$, one finds that there is no peak in $c_{\mathrm{V}} / T$ before the crossover

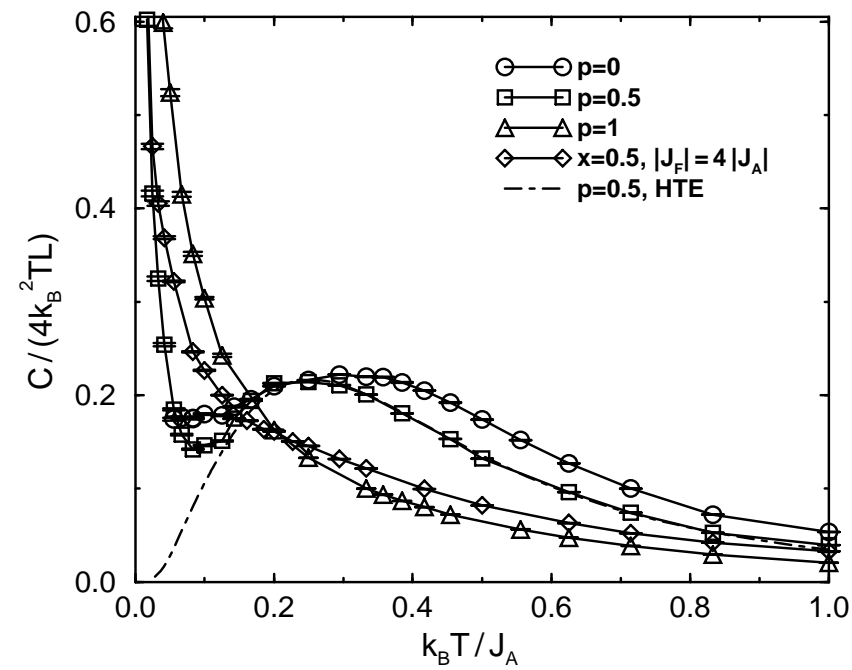

Fig. 5. The specific heat per spin $c_{\mathrm{V}}$ divided by the temperature $T$ for the uniform and random bond systems. The area below the curves corresponds to the entropy $S$. The symbols are the QMC results, the dot-dashed line (HTE) is the high-temperature expansion result obtained by the extrapolation of the two-point Padé approximants. One can see that the QMC and hightemperature expansion results are in perfect agreement down to $k_{\mathrm{B}} T \approx 0.2 J$, then the QMC results show the correct crossover to a divergent behavior.

to the divergent low-temperature behavior as for the unconstrained model, but $c_{\mathrm{V}} / T$ is rather continuously increasing for $T \rightarrow 0$.

\section{$\S 6 . \quad$ Conclusions}

We have investigated numerically the thermodynamic properties of one dimensional spin chains with random FM or AF couplings. We have considered an unconstrained model with equal magnitude of the $\mathrm{AF}$ and FM couplings and a realistic model of $\mathrm{Sr}_{3} \mathrm{CuPt}_{1-x} \mathrm{Ir}_{x} \mathrm{O}_{6}$ (constrained model). The thermodynamic properties of the random bond systems are determined by three different energy scales: at high temperatures, the energy scale is given by the original spin-spin couplings. The susceptibility obeys a Curie law of free spins at very high temperatures $\left(T \gg k_{\mathrm{B}} T\right)$. By lowering the temperature, the spins start to correlate within the AF and FM segments and form effective spins. At intermediate temperatures, these effective spins interact very weakly, essentially as free spins. The crossover of the individual spins to effective spin segments can be seen by a peak in the specific heat and a Curie-law of the magnetic susceptibility of the effective spins. The energy scale of this intermediate temperature regime is given by the interactions of these effective spins. At still lower temperatures, the interactions among the effective spins become relevant and the spin segments gradually freeze out into clusters of correlated spin segments. Also this crossover to the lowtemperature scaling regime is visible by a peak in the specific heat, although this peak is much smaller than 
for the previous transition.

Our results represent the first exact treatment of the original Hamiltonian showing a clear separation into three different temperature regimes for the unconstrained model. This separation can be seen by two marked peaks in the specific heat and two Curie laws in the uniform magnetic susceptibility at high and intermediate temperatures. In order to allow the comparison with experimental results, we investigate for the first time a realistic model of $\mathrm{Sr}_{3} \mathrm{CuPt}_{1-x} \mathrm{Ir}_{x} \mathrm{O}_{6}$ with a constrained distribution of the FM bonds exactly.

We find that the three energy levels are not so clearly separated in this case. Because of the different magnitude of the FM and AF couplings, the energy scales are overlapping and the crossover between the three regimes is continuous. Therefore the peak originating from the correlation of the original spins is very broad and the onset of correlations among the effective spins can only be seen by a small cusp in the specific heat. Instead of a clear plateau in $\chi T$, the effective spins form a broad peak.

Overlapping energy-scales can be found in many random bond systems, indeed, the formation of a clearly distinguishable intermediate temperature regime depends subtly on the distribution and strength of the FM/AF bonds. However, the general picture still applies also for systems with overlapping energy scales and we have given upper and lower bounds for the Curie-constant of the susceptibility in the intermediate temperature regime of the constrained model. The calculation of these Curieconstants can be used as a universal tool for the analysis of the bond-distribution, e.g. much larger Curieconstants will result in experiments if the distribution of Pt- or Ir-ions is not really random because of large FM clusters which can enhance the Curie-constant drastically at low temperature. The calculation of these Curieconstants can also be applied in the statistical clusteranalysis of bond-distributions. 8.13)

We have given upper bounds for the beginning of the low-temperature scaling regime, $T=0.02 \mathrm{~J}$ for the generic model and $T=0.01 J_{\mathrm{A}}$ for the realistic model. But despite the very efficient QMC algorithm, we have not been able to reach the low-temperature scaling regime with the original Hamiltonian for both models. For the original Hamiltonian, the scaling regime starts at extremely low temperatures, such that a completely different approach will be needed to reach this regime with the original Hamiltonian. However, our numerical results confirm that the intermediate temperature regime is well described by an effective Hamiltonian for the effective spins, providing a firm basis for the investigations of the scaling regime based on the effective Hamiltonian.

We would like to finish by mentioning that an analogue of the random bond spin chains exists for very dilutely randomly depleted Heisenberg ladders.14) Due to the spin gap in the parent material, the Curie law of the effective spins can be observed experimentally more easily and the value of the Curie constant is in agreement with the theoretical value.15

\section{Acknowledgements}

We wish to thank B. Frischmuth, A. Furusaki, P.A. Lee, N. Nagaosa, K. Tanaka, and M. Troyer for helpful discussions. B.A. gratefully acknowledges support from an ETH-internal grant No. 9452/41-2511.5. All the calculations have been performed on the Intel Paragon XP/S-22 MP of ETH Zürich.

[1] J.P. Renard, M. Verdaguer, L. P. Regnault, W. A. C. Erkelens, J. Rossat-Mignod, J. Ribas, W. G. Stirling, and C. Vettier: J. Appl. Phys. 63 (1988) 3538; M. Date and K. Kindo: Phys. Rev. Lett. 65 (1990) 1659.

[2] Extended Linear Chain Compounds, edited by J. S. Miller (Plenum, New York, 1983), Vol. 3.

[3] A. P. Wilkinson and A. K. Cheetham: Acta Crystallogr. Sect. C 45 (1989) 1672; A. P. Wilkinson, A. K. Cheetham, W. Kunman, and A. Kvick: Eur. J. Solid State Inorg. Chem. 28 (1991) 453.

[4] T.N. Nguyen, P.A. Lee, and H.-C. zur Loye: Science 271 (1996) 489.

[5] A. Furusaki, M. Sigrist, P.A. Lee, K. Tanaka and N. Nagaosa: Phys. Rev. Lett. 73 (1994) 2622; A. Furusaki, M. Sigrist, E. Westerberg, P.A. Lee, K. Tanaka and N. Nagaosa: Phys. Rev. B 52 (1995) 15930.

[6] E. Westerberg, A. Furusaki, M. Sigrist and P.A. Lee: Phys. Rev. Lett. 75 (1995) 4302.

[7] E. Westerberg, A. Furusaki, M. Sigrist and P.A. Lee: Phys. Rev. B. 55 (1997) 12578.

[8] B. Frischmuth and M. Sigrist: Phys. Rev. Lett. 79 (1997) 147.

[9] H.G. Evertz, G. Lana, and M. Marcu: Phys. Rev. Lett. 70 (1993) 875 .

[10] H.F. Trotter: Proc. Am. Math. Soc. 10 (1959) 545; M. Suzuki: Prog. of Theor. Phys. 56 (1976) 1454.

[11] N. Metropolis, A.R. Rosenbluth, M.N. Rosenbluth, A.H. Teller and E. Teller: J. of Chem. Phys. 21 (1953) 1087.

[12] M. Sweeny: Phys. Rev. B 27 (1983) 4445; U. Wolff: Phys. Rev. Lett. 60 (1988) 1461; U. Wolff: Nucl. Phys. 300[FS22] (1988) 501.

[13] B. Frischmuth, M. Sigrist, B. Ammon, and M. Troyer: condmat/9808027.

[14] M. Sigrist and A. Furusaki: J. Phys. Soc. Jpn. 65 (1996) 2385; Y. Iino and M. Imada: J. Phys. Soc. Jpn. 65 (1996) 3728; T. Miyzaki, M. Troyer, M. Ogata, K. Ueda, and D. Yoshioka: J. Phys. Soc. Jpn. 66 (1997) 2580.

[15] M. Azuma, Y. Fujishiro, M. Takano, M. Nohara, and H. Takagi: Phys. Rev. 55 (1997) R8658. 